\title{
Compositional modelling of the impact of source lithology on the plant ionome
} RAIMON TOLOSANA-DELGADO', MAARIT MIDDLETON ${ }^{2}$

Helmholtz Zentrum Dresden-Rossendorf, Helmholtz Institute Freiberg for Resource Technology, Chemnitzerstrasse 40, 09599 Freiberg, Germany, s.pospiech@hzdr.de

Geologian tutkimuskeskus GTK, Environmental Geology, Lähteenie 2, 96101 Rovaniemi, Finland

The plant ionome is used for many applications for which it is important to understand and model how the elemental composition in the plants or plant organs evolved based on the available element sources, e.g. the effect of the soils or the underlying lithologies. Since the uptake and translocation of elements is influenced by a multitude of non-controllable parameters it is very challenging to relate a certain element pattern specifically to one parameter and quantify the effect of the parameter. Additionally, the applied modelling tools often do not take into account that both, the plant ionome and the element source, are multi-element concentrations. Concentrations are compositional data which represent the relative importance of some parts of a whole, and thus they are constrained and typically might suffer from problems of spurious correlations and negative bias, which disturbs our models of plant uptake.

In this contribution, we are presenting a statistical approach to describe the relation between geochemical composition of plants and the bedrock lithology while taking the multi-variate and constrained nature of concentrations into account. Modern compositional data analysis relies on logratio transforms and the Aitchison geometry. Most of the processes building the plant ionome from the element sources can be understood as linear modifications in the log-ratio space. A selective element transport would correspond to a shift of log-ratios. If this selectivity varies randomly this shift can be modelled by a multivariate normal distribution in logratio space. Selective element uptake by plants can thus be modelled by a downscaling of variability in the form of regression coefficients in log-ratio space. This allows to model many geochemical phenomena through a multivariate linear model in log-ratios.

To demonstrate the statistical method an exploration data set of lithologies and plant data from Northern Finland is used. 